\title{
ASYMPTOTIC BEHAVIOR OF A GENERAL CLASS OF MIXTURE FAILURE RATES
}

\author{
MAXIM FINKELSTEIN, ${ }^{*}$ University of the Free State, Bloemfontein, and \\ Max Planck Institute for Demographic Research \\ VERONICA ESAULOVA,** Otto-von-Guericke-Universität Magdeburg
}

\begin{abstract}
Mixtures of increasing failure rate distributions can decrease, at least in some intervals of time. Usually this property is observed asymptotically, as $t \rightarrow \infty$, which is due to the fact that a mixture failure rate is 'bent down', as the weakest populations are dying out first. We consider a survival model that generalizes additive hazards models, proportional hazards models, and accelerated life models very well known in reliability and survival analysis. We obtain new explicit asymptotic relations for a general setting and study specific cases. Under reasonable assumptions we prove that the asymptotic behavior of the mixture failure rate depends only on the behavior of the mixing distribution in the neighborhood of the left-hand endpoint of its support, and not on the whole mixing distribution.
\end{abstract}

Keywords: Mixture of distributions; decreasing failure rate; increasing failure rate; proportional hazards model; accelerated life model

2000 Mathematics Subject Classification: Primary 62E10; 60E15

\section{Introduction}

Mixtures of decreasing failure rate distributions are always decreasing failure rate (Barlow and Proschan (1975, pp. 101-104)). However, mixtures of increasing failure rate distributions can decrease, at least in some intervals of time, which means that the increasing failure rate class of distributions is not closed under the operation of mixing (Lynch (1999)). As increasing failure rate distributions usually model lifetimes governed by ageing processes, this implies that the operation of mixing can change the pattern of ageing, e.g. from positive ageing (increasing failure rate) to negative ageing (decreasing failure rate). These important facts should be taken into account in applications.

It is hard to find homogeneous populations in real life, and mixtures of distributions usually present an effective tool for modeling heterogeneity. A natural specific approach to this modeling exploits the notion of a nonnegative, random, unobserved parameter (frailty), $Z$, introduced by Vaupel et al. (1979) in a demographic context. This in fact leads to the consideration of a random failure rate, $\lambda(t, Z)$. As the failure rate is a conditional characteristic, the 'ordinary' expectation with respect to $Z, \mathrm{E}[\lambda(t, Z)]$, does not define a mixture failure rate, $\lambda_{\mathrm{m}}(t)$, and proper conditioning should be performed (Finkelstein (2004)).

Received 26 August 2005; revision received 7 January 2006.

* Postal address: Department of Mathematical Statistics, University of the Free State, PO Box 339, 9300 Bloemfontein, Republic of South Africa. Email address: finkelm.sci@mail.uovs.ac.za

** Postal address: Faculty of Mathematics, Otto-von-Guericke-Universität Magdeburg, Postfach 4120, D-39016 Magdeburg, Germany. Email address: veronica.esaulova@gmail.com 
A convincing 'experiment' showing the deceleration of the observed failure rate occurs in nature. It is well known that human mortality follows the Gompertz lifetime distribution with an exponentially increasing mortality rate. Assume that heterogeneity can be described by the proportional gamma frailty model

$$
\lambda(t, Z)=Z \alpha \mathrm{e}^{\beta t},
$$

where $\alpha$ and $\beta$ are positive constants defining a baseline mortality rate. Owing to its computational simplicity, the gamma frailty model is largely the only one so far used in applications. It can be shown (see, e.g. (25) below) that in this case the mixture failure rate, $\lambda_{\mathrm{m}}(t)$, is monotone on $[0, \infty]$ and tends to a constant as $t \rightarrow \infty$. It is monotone increasing, however, for the actual parameter values of this model. This fact explains the recently observed deceleration in mortality of the very oldest humans (the human mortality plateau; see Thatcher (1999)). A similar result was experimentally obtained for a large cohort of medflies by Carey et al. (1992). However, in engineering applications the operation of mixing can result in an even more dramatic effect: the mixture failure rate increases on $\left[0, t_{\mathrm{m}}\right), t_{\mathrm{m}}>0$, and decreases asymptotically to 0 on $\left[t_{\mathrm{m}}, \infty\right)$. This was experimentally observed by Finkelstein (2005) for a heterogeneous sample of miniature light bulbs. This fact is easily explained theoretically using the gamma frailty model with a baseline failure rate increasing, in accordance with a Weibull law, like a power function (Gupta and Gupta (1996), Finkelstein and Esaulova (2001a)).

In Block et al. (2003) it was proved that if the failure rate of each subpopulation converges to a constant, and this convergence is uniform, then the mixture failure rate converges to the failure rate of the strongest subpopulation: the weakest subpopulations die out first. (For convenience, from now on we shall use the term 'population' instead of 'subpopulation', where appropriate.) Those authors generalized a case with constant population failure rates considered by Clarotti and Spizzichino (1990) and presented a further development of Block et al. (1993) (see also Lynn and Singpurwalla (1997) and Gurland and Sethuraman (1995)). In Block and Joe (1997), the following asymptotic result, which also addresses the issue of ultimate monotonicity, was obtained. Let $z_{0}$ be a realization of a frailty, $Z$, which corresponds to the strongest population. If $\lambda(t, z) / \lambda\left(t, z_{0}\right)$ uniformly decreases as $t \rightarrow \infty$, then $\lambda_{\mathrm{m}}(t) / \lambda\left(t, z_{0}\right)$ also decreases. If, in addition, $\lim _{t \rightarrow \infty} \lambda\left(t, z_{0}\right)$ exists, then the quotient decreases to 1 .

The recent paper of Li (2005) generalized the results of Block et al. (2003) using similar analytical tools and approaches. Instead of assuming that each individual failure rate has a limit, the author assumed that there exists an asymptotic baseline function $\lambda(t)$ such that the ratio of each individual failure rate to the asymptotic baseline function $\lambda(t, z) / \lambda(t)$ has a limit. Li showed that, under certain conditions, the ratio of the mixture failure rate to the asymptotic baseline function has a limit. As in Block et al. (2003), it was shown that this limit is the corresponding essential infimum. The stringent condition of the uniform convergence of $\lambda(t, z) / \lambda(t)$ to some $a(z)$ was again imposed. Therefore, Li (2005) combines the analytical reasoning of Block et al. (2003) with the 'ratio approach' of Block and Joe (1997).

The models in the papers mentioned are in fact generalized proportional hazards models. They are based on the asymptotic equivalence $\lambda(t, z) \sim \lambda(t) a(z)$, in the sense of the uniform convergence of the ratio to 1 , which is, as already mentioned, a rather strong assumption. Note that the strongest population is not always identifiable.

Our approach is totally different. Our main focus is on explicit asymptotic formulae and proving the results linking the asymptotic behavior of mixture failure rates with the behavior of a mixing distribution in the neighborhood of the left-hand endpoint of its support. The goal of the current paper is to try to find a balance between the generality of a model and the possibility 
of obtaining explicit asymptotic results for the mixture failure rate, $\lambda_{\mathrm{m}}(t)$. We suggest a class of distribution that hopefully meets this requirement, and develop a new approach to this kind of application that is related to the theory and methods of generalized convolutions, e.g. Laplace and Fourier transforms, and especially Mellin convolutions (Bingham et al. (1987)). In proving our asymptotic results we use a standard technique similar to that used in obtaining Abelian, Tauberian, and Mercerian-type theorems, although our theorems are not direct corollaries of results in this field. In line with this relationship it turns out that the asymptotic behavior of mixture failure rates for the suggested class of lifetime distributions depends only on the behavior of the mixing distribution in the neighborhood of $\inf _{z}\{\pi(z)>0\}$, and not on the whole mixing probability density function (PDF) $\pi(z)$.

After defining the survival model in Section 2, we formulate our main theorems in Section 3 and consider examples important for applications in Section 4. As the proofs are more technical than we had hoped, they are deferred to Section 5. We conclude in Section 6.

\section{The survival model}

Let $T \geq 0$ be a lifetime random variable with cumulative density function (CDF) $F(t)$ (and let $\bar{F}(t)=1-F(t))$. Assume that $F(t)$ is indexed by a nonnegative random variable, $Z$, with a $\operatorname{PDF} \pi(z)$ and support on $[0, \infty)$ :

$$
\mathrm{P}(T \leq t \mid Z=z) \equiv \mathrm{P}(T \leq t \mid z)=F(t, z) .
$$

Also assume that the PDF $f(t, z)$ exists. The corresponding failure rate for every fixed $z$ is thus $\lambda(t, z)=f(t, z) / \bar{F}(t, z)$. The support $[a, b], a>0, b<\infty$, can also be considered. Thus, the mixture $\mathrm{CDF}$ and PDF are respectively defined by

$$
F_{\mathrm{m}}(t)=\int_{0}^{\infty} F(t, z) \pi(z) \mathrm{d} z, \quad f_{\mathrm{m}}(t)=\int_{0}^{\infty} f(t, z) \pi(z) \mathrm{d} z .
$$

The mixture failure rate is

$$
\lambda_{\mathrm{m}}(t)=\frac{f_{\mathrm{m}}(t)}{\bar{F}_{\mathrm{m}}(t)}=\frac{\int_{0}^{\infty} f(t, z) \pi(z) \mathrm{d} z}{\int_{0}^{\infty} \bar{F}(t, z) \pi(z) \mathrm{d} z} .
$$

As is usual, we denote the cumulative failure rate by

$$
\Lambda(t, z)=\int_{0}^{t} \lambda(u, z) \mathrm{d} u .
$$

We will define a class of lifetime distributions $F(t, z)$ and study the asymptotic behavior of the corresponding mixture failure rate, $\lambda_{\mathrm{m}}(t)$. It is more convenient now to give this definition in terms of the cumulative failure rate, $\Lambda(t, z)$, rather than in terms of the failure rate $\lambda(t, z)$. The basic model is given by a relation of the following form:

$$
\Lambda(t, z)=A(z \phi(t))+\psi(t) .
$$

\subsection{General assumptions on the basic model}

Natural properties of the cumulative failure rate of the absolutely continuous distribution $F(t, z), z \in[0, \infty)$, imply that the functions $A(s), \phi(t)$, and $\psi(t)$ are differentiable, that the right-hand side of (2) is nondecreasing in $t$ and tends to infinity as $t \rightarrow \infty$, and that 
$A(z \phi(0))+\psi(0)=0$. Therefore, these properties will be assumed throughout the paper, although some of them will not be needed in the formal proofs.

An important additional simplifying assumption is that $A(s), s \in[0, \infty)$, and $\phi(t), t \in$ $[0, \infty)$, are increasing functions of their arguments, although some generalizations (e.g. for ultimately increasing functions) can easily be made. Therefore, in this paper we will view $1-\mathrm{e}^{-A(z \phi(t))}, z \neq 0$, as a lifetime CDF.

It should be noted that model (2) can also be easily generalized to the form $\Lambda(t, z)=$ $A(g(z) \phi(t))+\psi(t)+\eta(z)$ for some properly defined functions $g(z)$ and $\eta(z)$. However, we cannot generalize further (at least at this stage), and the multiplicative form of the arguments in $A(g(z) \phi(t))$ is important for our method of deriving asymptotic relations. It is also clear that the additive term $\psi(t)$, although important in applications, gives only a slight generalization in further analysis of $\lambda_{\mathrm{m}}(t)$, as (2) can be interpreted in terms of two components in series (or, equivalently, via two competing risks). However, this term will be essential in Section 3, in defining the strongest population.

The failure rate corresponding to the cumulative failure rate $\Lambda(t, z)$ is

$$
\lambda(t, z)=z \phi^{\prime}(t) A^{\prime}(z \phi(t))+\psi(t) .
$$

We are now able to explain why we start with the cumulative failure rate rather than with the failure rate itself, as is often done in lifetime modeling. The reason is that we can easily suggest intuitive interpretations for (2), whereas it is certainly not so simple to interpret the failure rate structure of the form (3) without stating that it just follows from the structure of the cumulative failure rate.

Relation (2) defines a rather broad class of survival models that can be used, e.g. for modeling the impact of environment on characteristics of survival. The proportional hazards, additive hazards, and accelerated life models, which are widely used in reliability, survival analysis and risk analysis, are the obvious specific cases of (2) and (3). Then

(Multiplicative) proportional hazards model. Let $A(u) \equiv u, \phi(t)=\Lambda(t)$, and $\psi(t)=0$.

$$
\lambda(t, z)=z \lambda(t), \quad \Lambda(t, z)=z \Lambda(t) .
$$

Accelerated life model. Let $A(u) \equiv \Lambda(u), \phi(t)=t$, and $\psi(t)=0$. Then

$$
\lambda(t, z)=z \lambda(t z), \quad \Lambda(t, z)=\int_{0}^{t z} \lambda(u) \mathrm{d} u=\Lambda(t z) .
$$

Additive hazards model. Let $A(u) \equiv u$ and $\phi(t)=t$, and let $\psi(t)$ be increasing, with $\psi(0)=0$. Then

$$
\lambda(t, z)=z+\psi^{\prime}(t), \quad \Lambda(t, z)=z t+\psi(t) .
$$

The functions $\lambda(t)$ and $\psi^{\prime}(t)$ play the role of baseline failure rates in equations (5) and (6), respectively. Note that, in all these models, the functions $\phi(t)$ and $A(s)$ are monotone increasing.

The asymptotic behavior of mixture failure rates in the proportional hazards and additional hazards models was studied for some specific mixing distributions in, e.g. Gurland and Sethuraman (1995) and Finkelstein and Esaulova (2001a). However, as far as the authors know, the mixture failure rate for the accelerated life model has been considered at a descriptive level only, in Anderson and Louis (1995). 


\section{General results}

Our first theorem provides an asymptotic formula for the mixture failure rate, $\lambda_{\mathrm{m}}(t)$, under rather mild assumptions.

Theorem 1. Let the cumulative failure rate, $\Lambda(t, z)$, be given by model (2) and let the mixing $P D F \pi(z)$ be defined as

$$
\pi(z)=z^{\alpha} \pi_{1}(z),
$$

where $\alpha>-1$ and $\pi_{1}(z), \pi_{1}(0) \neq 0$, is a function bounded on $[0, \infty)$ and continuous at $z=0$.

Also assume that

$$
\phi(t) \rightarrow \infty \text { as } t \rightarrow \infty
$$

and

$$
\int_{0}^{\infty} \mathrm{e}^{-A(s)} s^{\alpha} \mathrm{d} s<\infty,
$$

where $A(s)$ is also ultimately increasing. Then

$$
\lambda_{\mathrm{m}}(t)-\psi^{\prime}(t) \sim(\alpha+1) \frac{\phi^{\prime}(t)}{\phi(t)} .
$$

In (10), by ' $\sim$ ' we as usual mean asymptotic equivalence, writing $a(t) \sim b(t)$ as $t \rightarrow \infty$ if $\lim _{t \rightarrow \infty} a(t) / b(t)=1$.

It is easy to see that assumption (7) holds for the main lifetime distributions, such as Weibull, gamma, lognormal, etc. Assumption (8) states a natural condition on the function $\phi(t)$, which can often be viewed as a scale transformation. Condition (9) ensures that the CDF, $1-\mathrm{e}^{-A(s)}$, not be 'too heavy tailed' (as, e.g. is the Pareto distribution $1-s^{-\beta}$, for $s \geq 1$ and $\beta-\alpha>1$ ), and in our assumptions is equivalent to the condition of existence of the moment of order $\alpha+1$ for this CDF. The examples in the next section will clearly show that these conditions are not at all stringent and can be easily met in most practical situations.

A crucial feature of this result is that the asymptotic behavior of the mixture failure rate (omitting an obvious additive term, $\psi(t)$ ) depends only on the behavior of the mixing distribution in the neighborhood of 0 and the derivative of the logarithm of the scale function $\phi(t)$, given by $(\log \phi(t))^{\prime}=\phi^{\prime}(t) / \phi(t)$. When $\pi(0) \neq 0$ and $\pi(z)$ is bounded on $[0, \infty)$, the result does not depend on the mixing distribution at all, as $\alpha=0$.

Theorem 1 (as well as Theorems 2 and 4 below) can be formally generalized to the case in which the mixing random variable, $Z$, does not necessarily possess an absolutely continuous $\mathrm{CDF}$ on $[0, \infty)$; it is sufficient that it should be absolutely continuous (from the right) at $z=0$.

We can formulate a more general result, which states a similar dependence on the behavior of the mixing distribution at 0 in terms of the asymptotic comparison of two mixture failure rates: if, under some assumptions, the two mixing distributions are equivalent at $z=0$, then the mixture failure rates are equivalent as $t \rightarrow \infty$.

Formally, we state this as follows.

Theorem 2. Let $f(t, z)$ and $\pi(z)$ respectively be the lifetime and mixing PDFs in the general mixing model (2). Assume that there exists a positive function, $\alpha(t)$, that is ultimately decreasing to 0 as $t \rightarrow \infty$ and satisfies

$$
\frac{\int_{0}^{\alpha(t)} f(t, z) \pi(z) d z}{\int_{0}^{\infty} f(t, z) \pi(z) d z} \rightarrow 1 .
$$


Denote another mixing PDF by $\rho(z)$ and assume that $\rho(z) / \pi(z)$ is bounded on $[0, \infty)$, continuous at 0 , and satisfies $\lim _{z \rightarrow 0} \rho(z) / \pi(z) \neq 0$. Then

$$
\lambda_{\mathrm{m}}^{\pi}(t):=\frac{\int_{0}^{\infty} f(t, z) \pi(z) \mathrm{d} z}{\int_{0}^{\infty} \bar{F}(t, z) \pi(z) \mathrm{d} z} \sim \frac{\int_{0}^{\infty} f(t, z) \rho(z) \mathrm{d} z}{\int_{0}^{\infty} \bar{F}(t, z) \rho(z) \mathrm{d} z}=: \lambda_{\mathrm{m}}^{\rho}(t)
$$

as $t \rightarrow \infty$.

It is worth noting that if $\psi \equiv 0$ and all other conditions of Theorem 1 hold, then (8) guarantees assumption (11) to hold.

It is important that when applying Theorem 2 we do not need a specific form of survival model. As will be seen from the theorem's proof, $\pi(z)$ and $\rho(z)$ also need not necessarily be probability density functions (local integrability is in fact sufficient). The following corollary exploits the latter fact in the case with $\pi(z) \equiv 1$.

Corollary 1. Let $f(x, t)$ be a lifetime PDF in the general mixing model (2). Assume that there exists a positive function, $\alpha(t)$, that is ultimately decreasing to 0 as $t \rightarrow \infty$ and satisfies

$$
\frac{\int_{0}^{\alpha(t)} f(t, z) \mathrm{d} z}{\int_{0}^{\infty} f(t, z) \mathrm{d} z} \rightarrow 1
$$

Let $\rho(z)$ be a positive function bounded on $[0, \infty)$, continuous at 0 , and satisfying $\rho(0) \neq 0$. Then

$$
\frac{\int_{0}^{\infty} f(t, z) \rho(z) \mathrm{d} z}{\int_{0}^{\infty} \bar{F}(t, z) \rho(z) \mathrm{d} z} \sim \frac{\int_{0}^{\infty} f(t, z) \mathrm{d} z}{\int_{0}^{\infty} \bar{F}(t, z) \mathrm{d} z}
$$

as $t \rightarrow \infty$.

Theorems 1 and 2 treat the case in which the support of the mixing distribution includes 0 , i.e. $z \in[0, \infty)$. If the support excludes 0 , the situation changes significantly and we observe the well-known principle that the mixture failure rate tends to the failure rate of the strongest population (Block and Joe (1997), Block et al. (2003), Finkelstein and Esaulova (2001a)).

Theorem 3. Let the class of lifetime distributions be defined by (2), where $\phi(t) \rightarrow \infty$ and $A(s)$ is twice differentiable. Assume that, as $s \rightarrow \infty$,

$$
\frac{A^{\prime \prime}(s)}{A^{\prime}(s)^{2}} \rightarrow 0
$$

and

$$
s A^{\prime}(s) \rightarrow \infty
$$

Also assume that, for all $b$ and $c, b, c>0, b<c$, the quotient $A^{\prime}(b s) / A^{\prime}(c s)$ is bounded as $s \rightarrow \infty$. Let the mixing PDF $\pi(z)$ be defined on $[a, \infty), a>0$, be bounded on this interval, be continuous at $z=a$, and satisfy $\pi(a) \neq 0$. Then

$$
\lambda_{\mathrm{m}}(t)-\psi^{\prime}(t) \sim a \phi^{\prime}(t) A^{\prime}(a \phi(t)) .
$$

It is clear that conditions (13) and (14) trivially hold for the specific multiplicative and additive models of the previous section. We will discuss them within the framework of the accelerated life model later. More generally, these conditions hold if $A(s)$ belongs to a class of functions of smooth variation (Bingham et al. (1987, p. 44)). 
Assume additionally that the family of failure rates (3) is ordered in $z$, at least ultimately:

$$
\lambda\left(t, z_{1}\right)<\lambda\left(t, z_{2}\right), \quad \text { for all } z_{1}, z_{2} \in\left[z_{0}, \infty\right) \text { with } z_{1}<z_{2} \text { and } z_{0} \geq 0 \text {, and } t \geq 0 .
$$

Then, as we mentioned, Theorem 3 can be interpreted via the principle that the mixture failure rate converges to the failure rate of the strongest population. (Note that in this case the righthand side of (15) can also be interpreted as the failure rate of the strongest population for a survival model defined by a random variable with the CDF $1-\mathrm{e}^{-A(z \phi(t))}$.) An interesting question arises: is this principle a 'universal law' or a consequence of the sufficient assumptions of Theorem 3? Theorem 1 gives us an idea of how to create counterexamples.

Example 1. Assume that all the conditions of Theorem 1 hold and, additionally, that $A^{\prime}(s)$ is increasing on $[0, \infty)$. Then an ordering of failure rates with respect to $z$ in family (3) holds (for each fixed $t>0$ ), resulting formally in the strongest population being defined as having $\lambda(t, 0)=\phi^{\prime}(t)$. Note, however, that $1-\mathrm{e}^{-A(z \phi(t))}, z=0$, cannot be viewed as a CDF. Therefore, the principle in question implies that $\lambda_{\mathrm{m}}(t) \sim \psi^{\prime}(t)$. Yet it follows from (10) that

$$
\lambda_{\mathrm{m}}(t) \sim \psi^{\prime}(t)+(\alpha+1)(\log \phi(t))^{\prime},
$$

and if the second term on the right-hand side of this relation increases faster than $\psi^{\prime}(t)$ as $t \rightarrow \infty$, then this term defines the asymptotic behavior of $\lambda_{\mathrm{m}}(t)$. It is clear that this is possible for rapidly increasing functions such as, e.g. $\exp \left\{t^{n}\right\}, n \geq 1$. Thus, if then $\psi^{\prime}(t)=o\left((\log \phi(t))^{\prime}\right)$, we have

$$
\lambda_{\mathrm{m}}(t) \sim(\alpha+1)(\log \phi(t))^{\prime},
$$

whereas the principle holds only when $(\log \phi(t))^{\prime}=o\left(\psi^{\prime}(t)\right)$.

\section{Specific models}

\section{1. (Multiplicative) proportional hazards model}

In conventional notation the baseline failure rate is usually denoted as $\lambda_{0}(t)$ (or $\left.\lambda_{\mathrm{b}}(t)\right)$. Therefore, model (4) reads

$$
\lambda(t, z)=z \lambda_{0}(t), \quad \Lambda_{0}(t)=\int_{0}^{t} \lambda_{0}(u) \mathrm{d} u,
$$

and the mixture failure rate is given by

$$
\lambda_{\mathrm{m}}(t)=\frac{\int_{0}^{\infty} z \lambda_{0}(t) \mathrm{e}^{-z \Lambda_{0}(t)} \pi(z) \mathrm{d} z}{\int_{0}^{\infty} \mathrm{e}^{-z \Lambda_{0}(t)} \pi(z) \mathrm{d} z} .
$$

As $A(u) \equiv u, \phi(t)=\Lambda_{0}(t)$, and $\psi(t) \equiv 0$ in this specific case, Theorems 1 and 3 simplify to the following corollaries.

Corollary 2. Assume that the mixing $\operatorname{PDF} \pi(z), z \in[0, \infty)$, can be written as

$$
\pi(z)=z^{\alpha} \pi_{1}(z)
$$

where $\alpha>-1$ and $\pi_{1}(z)$ is bounded on $[0, \infty)$, continuous at $z=0$, and satisfies $\pi_{1}(0) \neq 0$. Then the mixture failure rate for the multiplicative model (16) has the following asymptotic behavior:

$$
\lambda_{\mathrm{m}}(t) \sim \frac{(\alpha+1) \lambda_{0}(t)}{\int_{0}^{t} \lambda_{0}(u) \mathrm{d} u} .
$$


Corollary 3. Assume that the mixing PDF $\pi(z), z \in[a, \infty)$ (we can define $\pi(z)=0$ for $z \in[0, a)$ ), is bounded, right semicontinuous at $z=a$, and satisfies $\pi(a) \neq 0$. Then, in accordance with (15), the mixture failure rate for the model (16) has the following asymptotic behavior:

$$
\lambda_{\mathrm{m}}(t) \sim a \lambda_{0}(t) .
$$

Corollary 2 states a remarkable fact: the asymptotic behavior of the mixture failure rate, $\lambda_{\mathrm{m}}(t)$, depends only on the behavior of the mixing PDF in the neighborhood of $z=0$ and the baseline failure rate $\lambda_{0}(t)$. Corollary 3 describes the convergence of a mixture failure rate to the mixture failure rate of the strongest population. In this simple multiplicative case, the family of the failure rates is trivially ordered in $z$ and the strongest population has the failure rate $a \lambda_{0}(t)$.

The next theorem generalizes the result of Corollary 3.

Theorem 4. Assume that the mixing PDF $\pi(z)$ in model (16) has support on $[a, b], a<0$, $b \leq \infty$, and, for $z \geq a$, can be defined as

$$
\pi(z)=(z-a)^{\alpha} \pi_{1}(z-a),
$$

where $\alpha>-1$ and $\pi_{1}(z)$ is bounded on $[0, b-a]$, with $\pi_{1}(0) \neq 0$. Then

$$
\lambda_{\mathrm{m}}(t) \sim a \lambda_{0}(t)
$$

It is quite remarkable that the asymptotic result in this theorem does not depend on a mixing distribution even when there is a singularity at $z=a$. This differs from the case with $a=0$, in Corollary 2. Relation (21) also describes the convergence to the failure rate of the strongest population, which differs dramatically from the convergence described by (19). The explanation of this difference is quite obvious: owing to the multiplicative nature of the model, the behavior of $z \lambda_{0}(t)$ in the neighborhood of $z=0$ (for the PDF (18)) is different from the behavior of this product in the neighborhood of $z=a$ (for the PDF (20)).

The mixture failure rate given by (16) can be obtained explicitly when the Laplace transform of the mixing PDF, $\tilde{\pi}(z)$, is easily computed, as in Example 2 below. As the cumulative failure rate is monotone increasing in $t$, the mixture survival function is written in terms of the Laplace transform as

$$
\int_{0}^{\infty} \mathrm{e}^{-z \Lambda_{0}(t)} \pi(z) \mathrm{d} z=\tilde{\pi}\left(\Lambda_{0}(t)\right) .
$$

Therefore, (17) becomes

$$
\lambda_{\mathrm{m}}(t)=-\frac{\left(\tilde{\pi}\left(\Lambda_{0}(t)\right)\right)^{\prime}}{\tilde{\pi}\left(\Lambda_{0}(t)\right)}=-\left(\log \tilde{\pi}\left(\Lambda_{0}(t)\right)\right)^{\prime}
$$

and the corresponding inverse problem, namely to obtain the baseline failure rate, given the mixture failure rate and the mixing distribution, can also be solved (Finkelstein and Esaulova (2001b)).

Example 2. Let the mixing distribution be the gamma distribution with PDF

$$
\pi(z)=\left(\frac{z}{b}\right)^{c-1} \mathrm{e}^{-z / b} \frac{1}{b \Gamma(c)},
$$


where $b, c>0$. The Laplace transform of $\pi(z)$ is $\tilde{\pi}(t)=(t b+1)^{-c}$ and, therefore, the mixture failure rate is given by

$$
\lambda_{\mathrm{m}}(t)=\frac{b c \lambda_{0}(t)}{1+b \int_{0}^{t} \lambda_{0}(u) \mathrm{d} u} .
$$

The expected value of a random variable $Z$ with PDF (22) is $b c$, and the variance is $b^{2} c$. Thus, for the fixed expectation $\mathrm{E}[Z]=1$, the variance is $\sigma^{2}=b$ and (23) becomes

$$
\lambda_{\mathrm{m}}(t)=\frac{\lambda_{0}(t)}{1+\sigma^{2} \int_{0}^{t} \lambda_{0}(u) \mathrm{d} u},
$$

which first appeared in Vaupel et al. (1979) in the context of demographics. This form allows us to compare different mixtures for a fixed baseline distribution. We can see that, when the variance of the mixing distribution increases, the mixture failure rate decreases.

Obviously, the asymptotic behavior of $\lambda_{\mathrm{m}}(t)$ can be explicitly analyzed. We consider two specific cases. First, if the baseline distribution is Weibull with $\lambda_{0}=\lambda t^{\beta}$, then the mixture failure rate (23) is (see Gupta and Gupta (1996))

$$
\lambda_{\mathrm{m}}(t)=\frac{(\beta+1) \lambda b c t^{\beta}}{\beta+1+\lambda b t^{\beta+1}},
$$

which converges to 0 as $t \rightarrow \infty$ and satisfies $\lambda_{\mathrm{m}}(t) \sim(\beta+1) c t^{-1}$, exactly as prescribed by (19) (here $c=\alpha+1)$. Second, if the baseline distribution is Gompertz with $\lambda_{0}(t)=\mu \mathrm{e}^{\beta t}$, then simple transformations result in

$$
\lambda_{\mathrm{m}}(t)=\frac{\beta c \mathrm{e}^{\beta t}}{\mathrm{e}^{\beta t}+\beta / \mu b-1} .
$$

If $b=\beta / \mu$ then $\lambda_{\mathrm{m}}(t) \equiv \beta c$, if $b>\beta / \mu$ then $\lambda_{\mathrm{m}}(t)$ increases to $\beta / \mu$, and if $b<\beta / \mu$ then $\lambda_{\mathrm{m}}(t)$ decreases to $\beta / \mu$.

Let us return to a discussion of the convergence of the mixture failure rate to the failure rate of the strongest population in Example 1. It is reasonable to compare the asymptotic behaviors in (24) and (25) for the same mixing distribution (22). For the Weibull CDF, the mixture failure rate converges to 0 . This means that within the framework of the multiplicative model (16), where the family of failure rates is ordered in $z$, we still can speak in terms of convergence to the failure rate of the strongest population, defining the case with $z=0$ as having some 'generalized' (or formal) strongest failure rate, $\lambda(t, 0)=0$. As was mentioned, $1-\mathrm{e}^{-A(z \phi(t))}$ cannot be viewed as a CDF in this case, and this formally violates the general assumptions we made in Section 2. However, the failure rate for a Gompertz CDF does not converge to 0 ; rather, it converges to a constant, thus violating the principle of convergence to the failure rate of the strongest population, even formulated in a 'generalized' way. The reason - which accords with our discussion in Example 1 - is the rapid increase of the function $\phi(t)$, which is proportional to $\mathrm{e}^{\beta t}$ in the case of a Gompertz CDF.

\subsection{Accelerated life model}

In conventional notation this model is written as

$$
\lambda(t, z)=z \lambda_{0}(t z), \quad \Lambda(t, z)=\Lambda_{0}(t z)=\int_{0}^{t z} \lambda_{0}(u) \mathrm{d} u .
$$


Although the definition of the accelerated life model is also very simple, the presence of a mixing parameter, $z$, in the arguments makes analysis of the mixture failure rate more complex than in the multiplicative case. Therefore, as was mentioned, this model has not been quantitatively studied before. The mixture failure rate in this specific case is

$$
\lambda_{\mathrm{m}}(t)=\frac{\int_{0}^{\infty} z \lambda_{0}(t z) \mathrm{e}^{-\Lambda_{0}(t z)} \pi(z) \mathrm{d} z}{\int_{0}^{\infty} \mathrm{e}^{-\Lambda_{0}(t z)} \pi(z) \mathrm{d} z} .
$$

The asymptotic behavior of $\lambda_{\mathrm{m}}(t)$ can be described as a specific case of Theorem 1 with $A(s)=\Lambda_{0}(s), \phi(t)=t$, and $\psi(t) \equiv 0$.

Corollary 4. Assume that the mixing PDF $\pi(z), z \in[0, \infty)$, can be defined as $\pi(z)=$ $z^{\alpha} \pi_{1}(z)$, where $\alpha>1$ and $\pi_{1}(z)$ is continuous at $z=0$ and bounded on $[0, \infty)$, with $\pi_{1}(0) \neq 0$. Let the baseline distribution with the cumulative failure rate $\Lambda_{0}(t)$ have a moment of order $\alpha+1$. Then

$$
\lambda_{\mathrm{m}}(t) \sim \frac{\alpha+1}{t}
$$

as $t \rightarrow \infty$.

The conditions of Corollary 4 are not very strong and are relatively natural. Most of the widely used lifetime distributions have moments to all orders. The Pareto distribution will be discussed in the next example. As was stated, the conditions on the mixing distribution hold for, e.g. the Gamma and the Weibull distributions, which are commonly used as mixing distributions.

Note that (27) is a surprising result, at least at first sight, as it does not depend on the baseline distribution. It is also dramatically different from the multiplicative case (19). It follows from Example 2 that the asymptotic results coincide in the case of the Weibull baseline distribution; this should be obvious, as only for the Weibull distribution can the accelerated life model be reparameterized to obtain a proportional hazards model, and vice versa.

The following example shows other possibilities for the asymptotic behavior of $\lambda_{\mathrm{m}}(t)$ when one of the conditions of Corollary 4 does not hold.

Example 3. Consider the gamma mixing distribution $\pi(z)=z^{\alpha} \mathrm{e}^{-x} / \Gamma(\alpha+1)$. Let the baseline distribution be the Pareto distribution with density $f_{0}(t)=\beta / t^{\beta+1}, t \geq 1, \beta>0$.

For $\beta>\alpha+1$ both the conditions of Corollary 2 and (27) hold. Let $\beta \leq \alpha+1$, which means that the baseline distribution does not have an $(\alpha+1)$ th moment. Then one of the conditions of Corollary 4 is violated. In this case, it can be shown by direct derivation (see Section 5) that $\lambda_{\mathrm{m}}(t) \sim \beta / t$ as $t \rightarrow \infty$, whereas, for the general case,

$$
\lambda_{\mathrm{m}}(t) \sim \frac{\min (\beta, \alpha+1)}{t} .
$$

It can be shown that the same asymptotic relation holds not only for the gamma distribution, but also for any other mixing distribution of the form $\pi(z)=z^{\alpha} \pi_{1}(z)$. If $\beta>\alpha+1$ then the function $\pi_{1}(z)$ should be bounded, with $\pi_{1}(0) \neq 0$.

As $A(s)=\Lambda_{0}(s)$ and $\phi(t)=t$, Theorem 3 simplifies as follows.

Corollary 5. Assume that the mixing $\operatorname{PDF} \pi(z), z \in[a, \infty)$, is bounded, continuous at $z=a$, and satisfies $\pi(a) \neq 0$. Let

$$
\frac{\lambda_{0}^{\prime}(t)}{\lambda_{0}(t)^{2}} \rightarrow 0, \quad t \lambda_{0}(t) \rightarrow \infty
$$


as $t \rightarrow \infty$. Also assume that, for all $b$ and $c, b, c>0, b<c$, the quotient $\lambda_{0}(b x) / \lambda_{0}(c x)$ is bounded as $x \rightarrow \infty$. Then, in accordance with (15), the mixture failure rate for the model (26) has the following asymptotic behavior:

$$
\lambda_{\mathrm{m}}(t)-\psi^{\prime}(t) \sim a \lambda_{0}(a t) .
$$

Conditions (28) are rather weak. For example, in the marginal case of the Pareto distribution (with baseline failure rate of the form $\lambda_{0}(t)=c t^{-1}, c>0, t \geq 1$ ) (28) is not satisfied, but in mixing we are primarily interested in baseline failure rates that increase, at least ultimately.

Owing to its simplicity, the asymptotic behavior of $\lambda_{\mathrm{m}}(t)$ in the additive hazards model (6) does not deserve special attention. As $A(s) \equiv s$ and $\phi(t) \equiv t$, conditions (8) and (9) of Theorem 1, for instance, hold and the asymptotic result (10) simplifies to

$$
\lambda_{\mathrm{m}}(t)-\psi^{\prime}(t) \sim \frac{\alpha+1}{t} .
$$

\section{Proofs}

\subsection{Proof of Theorem 1}

We first need a simple lemma about Dirac sequences of functions.

Lemma 1. Let $g(z)$ and $h(z)$ be nonnegative, locally integrable functions on $[0, \infty)$ such that

$$
\int_{0}^{\infty} g(z) \mathrm{d} z<\infty
$$

and $h(z)$ is bounded and continuous at $z=0$. Then, as $t \rightarrow \infty$,

$$
t \int_{0}^{\infty} g(t z) h(z) \mathrm{d} z \rightarrow h(0) \int_{0}^{\infty} g(z) \mathrm{d} z .
$$

Proof. By substituting $u$ for $t z$, we have

$$
t \int_{0}^{\infty} g(t z) h(z) \mathrm{d} z=\int_{0}^{\infty} g(u) h(u / t) \mathrm{d} u .
$$

The function $h(u)$ is bounded and $h(u / t) \rightarrow 0$ as $t \rightarrow \infty$; thus, (29) holds by the dominated convergence theorem.

The proof of Theorem 1 is straightforward, using Definition (1) and Lemma 1.

The survival function for the model (2) is

$$
\bar{F}(t, z)=\mathrm{e}^{-A(z \phi(t))-\psi(t)} .
$$

By taking into account the fact that $\phi(t) \rightarrow \infty$ as $t \rightarrow \infty$, and applying Lemma 1 to the function $g(u)=\mathrm{e}^{-A(u)} u^{\alpha}$, we obtain

$$
\begin{aligned}
\int_{0}^{\infty} \bar{F}(t, z) \pi(z) \mathrm{d} z & =\int_{0}^{\infty} \mathrm{e}^{-A(z \phi(t))-\psi(t)} z^{\alpha} \pi_{1}(z) \mathrm{d} z \\
& \sim \frac{\mathrm{e}^{-\psi(t)} \pi_{1}(0)}{\phi(t)^{\alpha+1}} \int_{0}^{\infty} \mathrm{e}^{-A(s)} s^{\alpha} \mathrm{d} s
\end{aligned}
$$


where the integral is finite due to condition (9). The corresponding PDF is

$$
\begin{aligned}
f(t, z) & =\left(A^{\prime}(z \phi(t)) z \phi^{\prime}(t)+\psi^{\prime}(t)\right) \mathrm{e}^{-A(z \phi(t))-\psi(t)} \\
& =A^{\prime}(z \phi(t)) z \phi^{\prime}(t) \mathrm{e}^{-A(z \phi(t))-\psi(t)}+\psi^{\prime}(t) \bar{F}(t, z) .
\end{aligned}
$$

Similarly, by applying Lemma 1, we find that

$$
\begin{aligned}
\int_{0}^{\infty} & f(t, z) \pi(z) \mathrm{d} z-\psi^{\prime}(t) \int_{0}^{\infty} \bar{F}(t, z) \pi(z) \mathrm{d} z \\
& =\phi^{\prime}(t) \mathrm{e}^{-\psi(t)} \int_{0}^{\infty} A^{\prime}(z \phi(t)) \mathrm{e}^{-A(z \phi(t))} z^{\alpha+1} \pi_{1}(z) \mathrm{d} z \\
& \sim \frac{\phi^{\prime}(t) \mathrm{e}^{-\psi(t)} \pi_{1}(0)}{\phi(t)^{\alpha+2}} \int_{0}^{\infty} A^{\prime}(s) \mathrm{e}^{-A(s)} s^{\alpha+1} \mathrm{~d} s
\end{aligned}
$$

From condition (9) and the fact that $A(s)$ is ultimately increasing, we have

$$
\mathrm{e}^{-A(s)} s^{\alpha+1} \rightarrow 0 \quad \text { as } s \rightarrow \infty .
$$

Indeed, by the mean value theorem we have

$$
\int_{s}^{2 s} \mathrm{e}^{-A(u)} u^{\alpha} \mathrm{d} u=s \mathrm{e}^{-A\left(s_{1}\right)} s_{1}^{\alpha},
$$

for some $s_{1}, s \leq s_{1} \leq 2 s$. The right-hand side of this equation tends to 0 as $s$ tends to $\infty$. For $s$ larger than some $s_{0}$ we have $A\left(s_{1}\right)>A(s)$; thus, the left-hand side is smaller than $2^{\alpha} s^{\alpha+1} \mathrm{e}^{-A(s)}$, from which we recover (32). Using it while integrating by parts yields

$$
\int_{0}^{\infty} A^{\prime}(s) \mathrm{e}^{-A(s)} s^{\alpha+1} \mathrm{~d} s=(\alpha+1) \int_{0}^{\infty} \mathrm{e}^{-A(s)} s^{\alpha} \mathrm{d} s .
$$

Then, combining (30)-(33) finally gives

$$
\frac{\int_{0}^{\infty} f(t, z) \pi(z) \mathrm{d} z}{\int_{0}^{\infty} \bar{F}(t, z) \pi(z) \mathrm{d} z}-\psi^{\prime}(t) \sim(\alpha+1) \frac{\phi^{\prime}(t)}{\phi(t)} .
$$

\subsection{Proof of Theorem 2}

Lemma 2. Let $\{g(t, z), z \in[0, \infty)\}$ be a family of functions and $h(z)$ a single function satisfying the following conditions.

(i) For every $z \in[0, \infty)$ the function $g(t, z)$ is integrable in $t$, and for every $t \in[0, \infty)$ it is integrable in $z$.

(ii) There exists a function $\alpha(t)$ such that $\alpha(t) \rightarrow 0$ as $t \rightarrow \infty$ and

$$
\frac{\int_{0}^{\alpha(t)} g(t, z) \mathrm{d} z}{\int_{0}^{\infty} g(t, z) \mathrm{d} z} \rightarrow 1
$$

as $t \rightarrow \infty$.

(iii) The function $h(x)$ is locally integrable, bounded on $[0, \infty)$, and continuous at $z=0$. 
Then, as $t \rightarrow \infty$,

$$
\frac{\int_{0}^{\infty} g(t, z) h(z) \mathrm{d} z}{\int_{0}^{\infty} g(t, z) \mathrm{d} z} \rightarrow h(0)
$$

Proof. Let $h(z) \leq M, z \in[0, \infty)$, where $M$ is a positive constant. Then

$$
\frac{\int_{0}^{\infty} g(t, z) h(z) \mathrm{d} z}{\int_{0}^{\infty} g(t, z) \mathrm{d} z}=\frac{\int_{0}^{\alpha(t)} g(t, z) h(z) \mathrm{d} z}{\int_{0}^{\infty} g(t, z) \mathrm{d} z}+\frac{\int_{\alpha(t)}^{\infty} g(t, z) h(z) \mathrm{d} z}{\int_{0}^{\infty} g(t, z) \mathrm{d} z} .
$$

The second term is majorized by

$$
M \frac{\int_{\alpha(t)}^{\infty} g(t, z) \mathrm{d} z}{\int_{0}^{\infty} g(t, z) \mathrm{d} z}
$$

by condition (34). The first term converges to $h(0)$, due to the same condition and the fact that $h(z)$ is continuous at $z=0$.

To prove Theorem 2 we first show, in a direct way, that for $\bar{F}(t, z)$ there holds a condition similar to (11). For every $\varepsilon>0$, we choose a $t_{\varepsilon}$ such that, for $u>t_{\varepsilon}$, the function $\alpha(u)$ decreases and

$$
\int_{0}^{\alpha(u)} f(u, z) \pi(z) \mathrm{d} z>(1-\varepsilon) \int_{0}^{\infty} f(u, z) \pi(z) \mathrm{d} z .
$$

Since $\alpha(t)$ decreases, we have

$$
\int_{0}^{\alpha(t)} f(u, z) \pi(z) \mathrm{d} z>\int_{0}^{\alpha(u)} f(u, z) \pi(z) \mathrm{d} z
$$

for $u>t>t_{\varepsilon}$. Thus,

$$
\begin{aligned}
\int_{0}^{\alpha(t)} \bar{F}(t, z) \pi(z) \mathrm{d} z & =\int_{0}^{\alpha(t)} \int_{t}^{\infty} f(u, z) \mathrm{d} u \pi(z) \mathrm{d} z \\
& =\int_{t}^{\infty} \int_{0}^{\alpha(t)} f(u, z) \pi(z) \mathrm{d} z \mathrm{~d} u \\
& >\int_{t}^{\infty} \int_{0}^{\alpha(u)} f(u, z) \pi(z) \mathrm{d} z \mathrm{~d} u \\
& >(1-\varepsilon) \int_{t}^{\infty} \int_{0}^{\infty} f(u, z) \pi(z) \mathrm{d} z \mathrm{~d} u \\
& =(1-\varepsilon) \int_{0}^{\infty} \bar{F}(t, z) \pi(z) \mathrm{d} z .
\end{aligned}
$$

Now we apply Lemma 2 with $h(z)=\pi_{1}(z) / \pi(z)$ and $g(t, z)=f(t, z) \pi(z)$, which yields

$$
\frac{\int_{0}^{\infty} f(t, z) \rho(z) \mathrm{d} z}{\int_{0}^{\infty} f(t, z) \rho(z) \mathrm{d} z} \rightarrow h(0)
$$

Similarly, with $g(t, z)=\bar{F}(t, z) \pi(z)$ and the same $h(z)$, we have

$$
\frac{\int_{0}^{\infty} \bar{F}(t, z) \rho(z) \mathrm{d} z}{\int_{0}^{\infty} \bar{F}(t, z) \rho(z) \mathrm{d} z} \rightarrow h(0)
$$

as $t \rightarrow \infty$, and (12) follows immediately. 


\subsection{Proof of Theorem 3}

This theorem is rather technical and we must first prove three supplementary lemmas that present consecutive steps on the way to deriving the asymptotic relation (15).

Lemma 3. Let $h(x)$ be a twice-differentiable function with an ultimately positive derivative, such that

$$
\int_{0}^{\infty} \mathrm{e}^{-h(y)} \mathrm{d} y<\infty
$$

Also let $h^{\prime \prime}(x) / h^{\prime}(x)^{2} \rightarrow 0$ as $x \rightarrow \infty$. Then

$$
\int_{x}^{\infty} \mathrm{e}^{-h(y)} \mathrm{d} y \sim \mathrm{e}^{-h(x)} \frac{1}{h^{\prime}(x)}
$$

as $x \rightarrow \infty$.

Proof. Let $x_{0}$ be such that $h^{\prime}(x)>0$ for $x>x_{0}$. By (35), $h(x) \rightarrow \infty$ as $x \rightarrow \infty$. Thus, there exists an inverse function, $g(x)$, defined on $\left[x_{0}, \infty\right): g(h(x)):=h(g(x)) \equiv x$. The function $g(x)$ is also twice differentiable, and satisfies $g^{\prime}(x)=1 / h^{\prime}(g(x))$. Integrating by parts for $x>x_{0}$ yields

$$
\begin{aligned}
\int_{x}^{\infty} \mathrm{e}^{-h(y)} \mathrm{d} y & =\int_{h(x)}^{\infty} \mathrm{e}^{-u} g^{\prime}(u) \mathrm{d} u \\
& =\mathrm{e}^{-h(x)} g^{\prime}(h(x))+\int_{h(x)}^{\infty} \mathrm{e}^{-u} g^{\prime \prime}(u) \mathrm{d} u .
\end{aligned}
$$

Since

$$
\frac{g^{\prime \prime}(u)}{g^{\prime}(u)}=-\frac{h^{\prime \prime}(g(u))}{h^{\prime}(g(u))^{2}} \rightarrow 0
$$

as $u \rightarrow \infty$, the integral on the right-hand side of (36) vanishes in comparison with the one on the left-hand side. Therefore, after some simple algebra,

$$
\int_{x}^{\infty} \mathrm{e}^{-h(y)} \mathrm{d} y \sim \mathrm{e}^{-h(x)} g^{\prime}(h(x))=\mathrm{e}^{-h(x)} \frac{1}{h^{\prime}(x)} .
$$

Lemma 4. Let the assumptions of Lemma 3 hold. Assume additionally that, as $x \rightarrow \infty$,

$$
x h^{\prime}(x) \rightarrow \infty
$$

and that, for any $b$ and $c, b, c \geq a, b<c$, the quotient $h^{\prime}(b x) / h^{\prime}(c x)$ is bounded on $[0, \infty)$. Now let $\mu(u)$ be a positive, bounded, locally integrable function defined on $[a, \infty)$, continuous at $u=a$, and satisfying $\mu(a) \neq 0$. Then

$$
\int_{a}^{\infty} \mathrm{e}^{-h(u x)} \mu(u) \mathrm{d} u \sim \frac{\mu(a) \mathrm{e}^{-h(a x)}}{x h^{\prime}(a x)}
$$

as $x \rightarrow \infty$.

Proof. As the first step we prove that

$$
I(x):=\int_{a}^{\infty} \mathrm{e}^{-h(u x)} \mu(u) \mathrm{d} u \sim \mu(a) \int_{a}^{\infty} \mathrm{e}^{-h(u x)} \mathrm{d} u .
$$


As $\mu(u)$ is continuous at $u=a$, for $\varepsilon>0$ there exists a $\delta$ such that $|\mu(u)-\mu(a)|<\varepsilon$ if $|u-a|<\delta$. The function $\mu(u)$ is bounded; therefore, $\mu(u)<M$ for all $u \in[a, \infty)$ and for some positive $M>\mu(a)$. Thus, writing

$$
I(x)=\int_{a}^{a+\delta} \mathrm{e}^{-h(u x)} \mu(u) \mathrm{d} u+\int_{a+\delta}^{\infty} \mathrm{e}^{-h(u x)} \mu(u) \mathrm{d} u,
$$

we have

$$
\begin{aligned}
\left|I(x)-\mu(a) \int_{a}^{\infty} \mathrm{e}^{-h(u x)} \mathrm{d} u\right| & <\varepsilon \int_{a}^{a+\delta} \mathrm{e}^{-h(u x)} \mathrm{d} u+(M-\mu(a)) \int_{a+\delta}^{\infty} \mathrm{e}^{-h(u x)} \mathrm{d} u \\
& =\varepsilon \int_{a}^{\infty} \mathrm{e}^{-h(u x)} \mathrm{d} u+(M-\mu(a)-\varepsilon) \int_{a+\delta}^{\infty} \mathrm{e}^{-h(u x)} \mathrm{d} u,
\end{aligned}
$$

whence

$$
\left|\frac{I(x)}{\mu(a) \int_{a}^{\infty} \mathrm{e}^{-h(u x)} \mathrm{d} u}-1\right|<\frac{\varepsilon}{\mu(a)}+\frac{M-\mu(a)-\varepsilon}{\mu(a)} \frac{\int_{a+\delta}^{\infty} \mathrm{e}^{-h(u x)} \mathrm{d} u}{\int_{a}^{\infty} \mathrm{e}^{-h(u x)} \mathrm{d} u} .
$$

Lemma 3 yields

$$
\frac{\int_{a+\delta}^{\infty} \mathrm{e}^{-h(u x)} \mathrm{d} u}{\int_{a}^{\infty} \mathrm{e}^{-h(u x)} \mathrm{d} u}=\frac{\int_{a x+\delta x}^{\infty} \mathrm{e}^{-h(u)} \mathrm{d} u}{\int_{a x}^{\infty} \mathrm{e}^{-h(u)} \mathrm{d} u} \sim \frac{h^{\prime}(a x)}{h^{\prime}(a x+\delta x)} \mathrm{e}^{-(h(a x+\delta x)-h(a x))} .
$$

It follows from condition (37) and the mean value theorem that

$$
h(a x+\delta x)-h(a x)=\delta x h^{\prime}(s)>s h^{\prime}(s) \frac{\delta}{a+\delta}
$$

for some $s, a x<s<a x+\delta x$. Thus, $h(a x+\delta x)-h(a x) \rightarrow \infty$ as $x \rightarrow \infty$, the quotient $h^{\prime}(a x) / h^{\prime}(a x+\delta x)$ is bounded, and, therefore, the second summand on the right-hand side of (38) tends to 0 , whereas the first summand can be made arbitrarily small. This yields

$$
I(x) \sim \mu(a) \int_{a}^{\infty} \mathrm{e}^{-h(u x)} \mathrm{d} u
$$

as $x \rightarrow \infty$. Applying Lemma 3 completes the proof.

Lemma 5. Under the assumptions of Lemma 4, the following asymptotic relation holds as $x \rightarrow \infty$ :

$$
\int_{a}^{\infty} h^{\prime}(u x) \mathrm{e}^{-h(u x)} u \mu(u) \mathrm{d} u \sim \frac{a \mu(a)}{x} \mathrm{e}^{-h(a x)} .
$$

Proof. We first show that

$$
\int_{a}^{\infty} h^{\prime}(u x) \mathrm{e}^{-h(u x)} u \mathrm{~d} u \sim \frac{a}{x} \mathrm{e}^{-h(a x)} .
$$

Simple calculations give

$$
x^{2} \int_{a}^{\infty} h^{\prime}(u x) \mathrm{e}^{-h(u x)} u \mathrm{~d} u=\int_{a x}^{\infty} h^{\prime}(u) \mathrm{e}^{-h(u)} u \mathrm{~d} u=a x \mathrm{e}^{-h(a x)}+\int_{a x}^{\infty} \mathrm{e}^{-h(u)} \mathrm{d} u .
$$


By Lemma 4, we have

$$
\int_{a x}^{\infty} \mathrm{e}^{-h(u)} \mathrm{d} u \sim \mathrm{e}^{-h(a x)} \frac{1}{h^{\prime}(a x)} .
$$

We have assumed that $a x h^{\prime}(a x) \rightarrow \infty$ as $x \rightarrow \infty$; thus, $1 / h^{\prime}(a x)=o(a x)$ and

$$
x^{2} \int_{0}^{\infty} h^{\prime}(u x) \mathrm{e}^{-h(u x)} u \mathrm{~d} u \sim a x \mathrm{e}^{-h(a x)},
$$

which is the same as (40).

The next step is to prove that

$$
\int_{a}^{\infty} h^{\prime}(u x) \mathrm{e}^{-h(u x)} u \mu(u) \mathrm{d} u \sim \mu(a) \int_{a}^{\infty} h^{\prime}(u x) \mathrm{e}^{-h(u x)} u \mathrm{~d} u .
$$

We use the same $\varepsilon, \delta$, and $M$ as in Lemma 4, and similar reasoning, to obtain

$$
\left|\frac{\int_{a}^{\infty} h^{\prime}(u x) \mathrm{e}^{-h(u x)} u \mu(u) \mathrm{d} u}{\mu(a) \int_{a}^{\infty} h^{\prime}(u x) \mathrm{e}^{-h(u x)} u \mathrm{~d} u}-1\right|<\frac{\varepsilon}{\mu(a)}+\frac{\tilde{M}}{\mu(a)} \frac{\int_{a+\delta}^{\infty} h^{\prime}(u x) \mathrm{e}^{-h(u x)} u \mathrm{~d} u}{\int_{a}^{\infty} h^{\prime}(u x) \mathrm{e}^{-h(u x)} u \mathrm{~d} u},
$$

where $\tilde{M}=M-\mu(a)-\varepsilon$. By applying (40) and using (39) we obtain

$$
\frac{\int_{a+\delta}^{\infty} h^{\prime}(u x) \mathrm{e}^{-h(u x)} u \mathrm{~d} u}{\int_{a}^{\infty} h^{\prime}(u x) \mathrm{e}^{-h(u x)} u \mathrm{~d} u} \sim \frac{a+\delta}{a} \mathrm{e}^{-(h(a x+\delta x)-h(a x))} \rightarrow 0
$$

as $x \rightarrow \infty$. Again $\varepsilon / \mu(a)$ can be made arbitrarily small, which gives (41). Combining it with (40) completes the proof.

Now we are ready to prove Theorem 3 itself. Applying Lemma 4 as $t \rightarrow \infty$ results in

$$
\begin{aligned}
\int_{a}^{\infty} \bar{F}(t, z) \pi(z) \mathrm{d} z & =\int_{a}^{\infty} \mathrm{e}^{-A(z \phi(t))-\psi(t)} \pi(z) \mathrm{d} z \\
& =\mathrm{e}^{-\psi(t)} \int_{a}^{\infty} \mathrm{e}^{-A(z \phi(t))} \pi(z) \mathrm{d} z \\
& \sim \frac{\pi(a) \mathrm{e}^{-\psi(t)}}{\phi(t) A^{\prime}(a \phi(t))} \mathrm{e}^{-A(a \phi(t))} .
\end{aligned}
$$

In analogy to the corresponding step in the proof of Theorem 1, we have

$$
\begin{aligned}
\int_{a}^{\infty} & f(t, z) \pi(z) \mathrm{d} z-\psi^{\prime}(t) \int_{a}^{\infty} \bar{F}(t, z) \pi(z) \mathrm{d} z \\
& =\phi^{\prime}(t) \mathrm{e}^{-\psi(t)} \int_{a}^{\infty} A^{\prime}(z \phi(t)) \mathrm{e}^{-A(z \phi(t))} z \pi(z) \mathrm{d} z .
\end{aligned}
$$

Using Lemma 5 yields

$$
\int_{a}^{\infty} A^{\prime}(z \phi(t)) \mathrm{e}^{-A(z \phi(t))} z \pi(z) \mathrm{d} z \sim \frac{a \pi(a)}{\phi(t)} \mathrm{e}^{-A(a \phi(t))} .
$$


By combining the last three statements we arrive at (15):

$$
\begin{aligned}
\lambda_{\mathrm{m}}(t)-\psi^{\prime}(t) & =\frac{\int_{a}^{\infty} f(t, z) \pi(z) \mathrm{d} z}{\int_{a}^{\infty} \bar{F}(t, z) \pi(z) \mathrm{d} z}-\psi^{\prime}(t) \\
& \sim \frac{\phi^{\prime}(t) \mathrm{e}^{-\psi(t)} a \pi(a) \mathrm{e}^{-A(a \phi(t))}}{\phi(t)} \frac{A^{\prime}(a \phi(t)) \phi(t)}{\pi(a) \mathrm{e}^{-\psi(t)} \mathrm{e}^{-A(a \phi(t))}} \\
& =a \phi^{\prime}(t) A^{\prime}(a \phi(t)) .
\end{aligned}
$$

\subsection{Proof of Theorem 4}

As in Theorem 1, we consider the numerator and the denominator in (17) separately. By changing variables and applying Lemma 1 , we obtain

$$
\begin{aligned}
\int_{0}^{\infty} \bar{F}(t z) \pi(z) \mathrm{d} z & =\int_{a}^{\infty} \mathrm{e}^{-z \Lambda_{0}(t)}(z-a)^{\alpha} \pi_{1}(z-a) \mathrm{d} z \\
& =\mathrm{e}^{-a \Lambda_{0}(t)} \int_{0}^{\infty} \mathrm{e}^{-z \Lambda_{0}(t)} z^{\alpha} \pi_{1}(z) \mathrm{d} z \\
& \sim \frac{\mathrm{e}^{-a \Lambda_{0}(t)} \pi_{1}(0) \Gamma(\alpha+1)}{\Lambda_{0}(t)^{\alpha+1}}
\end{aligned}
$$

Similarly,

$$
\begin{aligned}
\int_{0}^{\infty} z f(t z) \pi(z) \mathrm{d} z= & \lambda_{0}(t) \int_{a}^{\infty} z \mathrm{e}^{-z \Lambda_{0}(t)}(z-a)^{\alpha} \pi_{1}(z-a) \mathrm{d} z \\
= & \lambda_{0}(t) \mathrm{e}^{-a \Lambda_{0}(t)} \int_{0}^{\infty} \mathrm{e}^{-z \Lambda_{0}(t)} z^{\alpha+1} \pi_{1}(z) \mathrm{d} z \\
& +a \lambda_{0}(t) \mathrm{e}^{-a \Lambda_{0}(t)} \int_{0}^{\infty} \mathrm{e}^{-z \Lambda_{0}(t)} z^{\alpha} \pi_{1}(z) \mathrm{d} z
\end{aligned}
$$

As $t \rightarrow \infty$, the first integral on the right-hand side is equivalent to $\pi_{1}(0) \Gamma(\alpha+2) \Lambda_{0}(t)^{-\alpha-2}$ and the second to $\pi_{1}(0) \Gamma(\alpha+1) \Lambda_{0}(t)^{-\alpha-1}$, which decreases more slowly than the first. Thus,

$$
\int_{0}^{\infty} z f(t z) \pi(z) \mathrm{d} z \sim a \pi_{1}(0) \Gamma(\alpha+1) \lambda_{0}(t) \frac{\mathrm{e}^{-a \Lambda_{0}(t)}}{\Lambda_{0}(t)^{\alpha+1}} .
$$

Finally, by using (42) and (43) in (17), we arrive at (21).

\subsection{Proof of Example 3}

By direct calculation, we obtain

$$
\begin{aligned}
\int_{0}^{\infty} f_{0}(t z) z \pi(z) \mathrm{d} z & =\int_{1 / t}^{\infty} \frac{\beta z}{t^{\beta+1} z^{\beta+1}} \frac{1}{\Gamma(\alpha+1)} \mathrm{e}^{-z} z^{\alpha} \mathrm{d} z \\
& =\frac{\beta}{\Gamma(\alpha+1) t^{\beta+1}} \int_{1 / t}^{\infty} z^{\alpha-\beta} \mathrm{e}^{-z} \mathrm{~d} z \\
& \sim \frac{\Gamma(\alpha-\beta+1) \beta}{\Gamma(\alpha+1) t^{\beta+1}}
\end{aligned}
$$

and

$$
\int_{0}^{\infty} \bar{F}_{0}(t z) \pi(z) \mathrm{d} z=\int_{0}^{1 / t} \frac{\mathrm{e}^{-z} z^{\alpha}}{\Gamma(\alpha+1)} \mathrm{d} z+\int_{1 / t}^{\infty} \frac{1}{t^{\beta} z^{\beta}} \frac{\mathrm{e}^{-z} z^{\alpha}}{\Gamma(\alpha+1)} \mathrm{d} z
$$


As $t \rightarrow \infty$ the first integral on the right-hand side is equivalent to

$$
\frac{1}{\Gamma(\alpha+1)} \int_{0}^{1 / t} z^{\alpha} \mathrm{d} z=\frac{1}{t^{\alpha+1} \Gamma(\alpha+2)}
$$

and the second is equivalent to $\Gamma(\alpha-\beta+1) / \Gamma(\alpha+1) t^{\beta}$, which for $\beta \leq \alpha$ decreases more slowly than the first; therefore, the sum of the two integrals is equivalent to $\Gamma(\alpha-\beta+1) / \Gamma(\alpha+1) t^{\beta}$. After some simple algebra, we obtain

$$
\lambda_{\mathrm{m}}(t) \sim \frac{\Gamma(\alpha-\beta+1) \beta}{\Gamma(\alpha+1) t^{\beta+1}} \frac{\Gamma(\alpha+1) t^{\beta}}{\Gamma(\alpha-\beta+1)}=\frac{\beta}{t} .
$$

If $\beta=\alpha+1$ then

$$
\int_{0}^{\infty} z f_{0}(t z) \pi(z) \mathrm{d} z=\frac{\alpha+1}{\Gamma(\alpha+1) t^{\alpha+2}} \int_{1 / t}^{\infty} z^{-1} \mathrm{e}^{-z} \mathrm{~d} z
$$

and, since

$$
\int_{0}^{1 / t} z^{\alpha} \mathrm{d} z=o\left(t^{-\alpha-1} \int_{1 / t}^{\infty} z^{-1} \mathrm{e}^{-z} \mathrm{~d} z\right)
$$

we obtain

$$
\int_{0}^{\infty} \bar{F}_{0}(t z) \pi(z) \mathrm{d} z \sim \int_{1 / t}^{\infty} \frac{1}{t^{\alpha+1} z} \frac{\mathrm{e}^{-z}}{\Gamma(\alpha+1)} \mathrm{d} z=\frac{1}{t^{\alpha+1} \Gamma(\alpha+1)} \int_{1 / t}^{\infty} z^{-1} \mathrm{e}^{-z} \mathrm{~d} z .
$$

Therefore,

$$
\lambda_{\mathrm{m}}(t) \sim \frac{\alpha+1}{t}=\frac{\beta}{t}
$$

\section{Concluding remarks}

Typically two types of result on mixture failure rate modeling have been considered in the literature: on the one hand the general asymptotic results of Clarotti and Spizzichino (1990), Block and Joe (1997), and Block et al. (1993), obtained under rather stringent conditions; and on the other hand specific proportional or additive hazards-type models of Gurland and Sethuraman (1995), Lynn and Singpurwalla (1997), and Finkelstein and Esaulova (2001a), to name a few, wherein some more detailed convergence properties have been described. It is worth noting, however, that the asymptotic behavior of the mixture failure rate in the accelerated life model has not been studied before, as the approaches used for proportional hazards and additive hazards models do not work in that case.

The survival model (2) of this paper generalizes all three conventional models and raises the possibility of deriving explicit asymptotic results. For example, Theorem 1 defines the asymptotic mixture failure rate in the case in which the mixing variable is defined on $[0, \infty)$, whereas Theorem 3 does so for $[a, \infty), a>0$.

Some of the results obtained can be generalized to a wider class of lifetime distribution than (2), but it seems as if the considered class is, in a way, 'optimal' in terms of the trade-off between the complexity of the model and the tractability (or applicability) of the results.

\section{Acknowledgement}

The authors are grateful to the anonymous referee for pointing out the recent paper of Y. Li (Li (2005)). 


\section{References}

Anderson, J. E. And Louis, T. A. (1995). Survival analysis using a scale change random effects model. J. Amer. Statist. Assoc. 90, 669-679.

Barlow, R. and Proschan, F. (1975). Statistical Theory of Reliability and Life Testing. Probability Models. Holt, Rinehart and Winston, New York.

Bingham, N. H., Goldie, C. M. And Teugels, J. L. (1987). Regular Variation. Cambridge University Press.

Block, H. W. And Joe, H. (1997). Tail behavior of the failure rate functions of mixtures. Lifetime Data Anal. 3, 269-288.

Block, H. W., LI, Y. And Savits, T. H. (2003). Initial and final behavior of failure rate functions for mixtures and systems. J. Appl. Prob. 40, 721-740.

Block, H. W., Mi, J. and Savits, T. H. (1993). Burn-in and mixed populations. J. Appl. Prob. 30, 692-702.

Carey, J. R., Liedo, P., Orozco, D. And Vaupel, J. W. (1992). Slowing of mortality rates at older ages in large medfly cohorts. Science 258, 457-461.

Clarotti, C. A. and Spizzichino, F. (1990). Bayes burn-in decision procedures. Prob. Eng. Inf. Sci. 4, 437-445.

Finkelstein, M. S. (2004). Minimal repair in heterogeneous populations. J. Appl. Prob. 41, 281-286.

Finkelstein, M. S. (2005). On some reliability approaches to human aging. Internat. J. Reliability Quality Safety Eng. 12, 1-10.

Finkelstein, M. S. And Esaulova, V. (2001a). Modeling a failure rate for a mixture of distribution functions. Prob. Eng. Inf. Sci. 15, 383-400.

Finkelstein, M. S. And Esaulova, V. (2001b). On an inverse problem in mixture hazard rates modelling. Appl. Stoch. Models Business Industry 17, 221-229.

Gupta, P. L. AND Gupta, R. C. (1996). Ageing characteristics of the Weibull mixtures. Prob. Eng. Inf. Sci. 10, 591-600.

Gurland, J. And Sethuraman, J. (1995). How pooling failure data may reverse increasing failure rate. J. Amer. Statist. Assoc. 90, 1416-1423.

LI, Y. (2005). Asymptotic baseline of the hazard rate function of mixtures. J. Appl. Prob. 42, 892-901.

LYNCH, J. D. (1999). On conditions for mixtures of increasing failure rate distributions to have an increasing failure rate. Prob. Eng. Inf. Sci. 13, 33-36.

Lynn, N. J. And SingPurwalla, N. D. (1997). Comment: "Burn-in” makes us feel good. Statist. Sci. 12, $13-19$.

Thatcher, A. R. (1999). The long-term pattern of adult mortality and the highest attained age. J. R. Statist. Soc. A 162, 5-43 (with discussion).

Vaupel, J. W., Manton, K. G. and Stallard, E. (1979). The impact of heterogeneity in individual frailty on the dynamics of mortality. Demography 16, 439-454. 